\title{
Increased MIR31HG IncRNA expression increases gefitinib resistance in non-small cell lung cancer cell lines through the EGFR/PI3K/AKT signaling pathway
}

\author{
BING WANG $^{1 *}$, HONG JIANG ${ }^{2 *}$, LIMIN WANG $^{3}$, XUEQIN CHEN $^{4}$, \\ $\mathrm{KAN}^{\mathrm{W}}{ }^{1}$, SHIRONG ZHANG ${ }^{1}$, SHENGLIN MA ${ }^{1}$ and BING XIA ${ }^{1}$ \\ ${ }^{1}$ Department of Radiation Oncology, Hangzhou First People's Hospital, \\ Hangzhou Cancer Hospital; Departments of ${ }^{2}$ Thoracic Surgery, ${ }^{3}$ Respiration and ${ }^{4}$ Medical Oncology, \\ Hangzhou First People's Hospital, Hangzhou, Zhejiang 310002, P.R. China
}

Received September 26, 2015; Accepted October 11, 2016

DOI: $10.3892 / \mathrm{ol} .2017 .5878$

\begin{abstract}
The aim of the present study was to gain insight into the molecular mechanism of gefitinib resistance in non-small cell lung cancer (NSCLC), and demonstrate whether long noncoding RNA (IncRNA) expression signatures differ between gefitinib-sensitive PC9 and gefitinib-resistant PC9 (PC9-R) cell lines. PC9 and PC9-R cells were treated with gefitinib and, after $48 \mathrm{~h}$, proliferation and apoptosis were analyzed using a Cell Counting Kit-8 (CCK-8) assay and flow cytometry. Microarray expression profiling of IncRNAs was undertaken in both PC9 and PC9-R cells, and the expression profiles were verified by reverse transcription quantitative-polymerase chain reaction. The EGFR/PI3K/AKT signaling pathway and mitochondrial apoptosis protein expression levels were assessed by western blot analysis. The PC9 cell line treated with gefitinib had a more significant effect on cell viability and apoptosis than the PC9-R cell line $(\mathrm{P}<0.05)$. Expression of various lncRNAs differed significantly between the two cell lines, and MIR31HG expression in particular was significantly higher in PC9-R cells. As expected, MIR31HG lncRNA knockdown sensitized PC9-R cells to gefitinib, and further experiments revealed that turning off the EGFR/PI3K/AKT signaling pathway activated expression of p53 in PC9-R cells transfected with si-MIR31HG. Furthermore, PC9-R cells transfected with si-MIR31HG induced cell apoptosis through the mitochondrial apoptosis pathway, and arrested the cell cycle in the G0/G1
\end{abstract}

Correspondence to: Professor Bing Xia, Department of Radiation Oncology, Hangzhou First People's Hospital, Hangzhou Cancer Hospital, 34 Yanguan Lane, Shangcheng, Hangzhou, Zhejiang 310002, P.R. China

E-mail: bxia_hzch@sina.com

${ }^{*}$ Contributed equally

Key words: MIR31HG long noncoding RNAs, EGFR/PI3K/AKT pathway, gefitinib resistance, non-small cell lung cancer phase. The results of the current study suggest that MIR31HG lncRNA levels in PC9-R cells are higher than in PC9 cells. Furthermore, overexpression of MIR31HG lncRNAs may contribute to gefitinib resistance in PC9-R cells through the EGFR/PI3K/AKT pathway, which impacts on cell proliferation, apoptosis and the cell cycle. MIR31HG IncRNA may therefore be a novel candidate biomarker for future therapeutic strategies involving EGFR-TKIs.

\section{Introduction}

Lung cancer is one of the leading causes of cancer-associated death, and non-small cell lung cancer (NSCLC) accounts for $\sim 80-85 \%$ of all lung cancer cases $(1,2)$. Gefitinib is an epidermal growth factor receptor tyrosine kinase inhibitor (EGFR-TKI) approved for first-line treatment of locally advanced or metastatic NSCLC with activating mutations of epidermal growth factor receptor (EGFR) tyrosine kinase (3). NSCLC initially exhibits an excellent response to gefitinib treatment (4). However, acquired resistance has been observed in many NSCLC patients after 6-12 months of treatment (5). Investigating the mechanism of resistance to EGFR-TKIs and identifying strategies capable of overcoming this resistance is thus an important clinical goal.

EGFR is overexpressed in NSCLC by $40-89 \%$ (6) and is one of the most important signaling components involved in cell growth and survival. As well as activating the phosphatidylinositol-3 kinase/protein kinase B (PI3K/AKT) signaling pathway and promoting tumor cell proliferation, EGFR overexpression may also decrease cell apoptosis by activating anti-apoptotic factors such as B-cell lymphoma 2-like protein 4 (Bax), Bcl-2-associated death promoter (Bad), and caspase-9, as well as inactivating pro-apoptotic transcription factors such as p53 (7).

The human transcriptome comprises large numbers of protein-coding messenger RNAs (mRNAs), and a large set of nonprotein coding transcripts, including long noncoding RNA (lncRNA) (8). lncRNAs are $>200$ nucleotides long, and their dysregulation appears to contribute to the growth and progression of human tumors $(9,10)$. Some studies have reported that 
dysregulated lncRNAs are related to lung cancer with lymph node metastasis, advanced stage lung cancer, metastasis development and poor patient prognosis (11-16). However, the mechanism by which changes in lncRNA levels affect the expression of gene products that may contribute to gefitinib resistance remains largely unknown.

The aim of the present study was to investigate gene expression profiling in the PC9 (formerly known as PC14) human non-small cell lung adenocarcinoma cell line. Microarray expression profiling of lncRNAs was undertaken in both PC9 cells and PC9 cells resistant to gefitinib (PC9-R). Expression levels of different lncRNAs in the two cell lines were examined, with the aim of revealing the mechanism by which PC9-R cells acquire resistance to gefitinib.

\section{Materials and methods}

Cell culture. The PC9 human non-small cell lung adenocarcinoma cell lines (formerly known as PC14) were obtained from the Cell Bank of the Chinese Academy of Sciences (Shanghai, China) and PC9 cells resistant to gefitinib (PC9-R) were routinely cultured in RPMI-1640 medium (Gibco-BRL; Thermo Fisher Scientific, Inc., Waltham, MA, USA) with $10 \%$ fetal bovine serum (FBS; Hyclone; GE Healthcare Life Sciences, Logan, UT, USA), $100 \mathrm{U} / \mathrm{ml}$ penicillin, $100 \mathrm{mg} / \mathrm{ml}$ streptomycin, and $1 \% \mathrm{~L}$-glutamine, and were maintained in a $5 \% \mathrm{CO}_{2}$ incubator at $37^{\circ} \mathrm{C}$ with saturated humidity. Subcultures were produced by trypsinization and were reseeded for experiments.

Cell proliferation assay. Cells were plated in 96-well flat-bottomed culture plates at a density of $5 \times 10^{3}$ cells/well. Various concentrations $(0.01,0.1,1,5,10,20,40$ and $80 \mu \mathrm{M})$ of gefitinib were then added to the plates. Following this, the media were replaced with RPMI-1640 and 1\% FBS (HyClone; GE Healthcare Life Sciences). The proliferative activity of cells after treatment with gefitinib was assessed using the Cell Counting Kit-8 (CCK-8; Dojindo Molecular Technologies, Inc., Kumamoto, Japan). Subsequent to incubation for $48 \mathrm{~h}$ in a $5 \% \mathrm{CO}_{2}$ humidified atmosphere at $37^{\circ} \mathrm{C}, 10 \mu \mathrm{l} \mathrm{CCK}-8$ reagent was added to each well, and the plates were incubated for a further $4 \mathrm{~h}$. Absorbance at $450 \mathrm{~nm}$ was determined spectrophotometrically using a microplate reader. Data were analyzed by the median-effect method to evaluate the drug concentrations that resulted in $50 \%$ growth inhibition $\left(\mathrm{IC}_{50}\right)$. The combination effect was evaluated by the CCK- 8 assay. Confidence interval values of $<1,1$ and $>1$ indicated synergism, additive effect and antagonism, respectively. Each treatment was assayed in triplicate during the same experiment.

Cell apoptosis assay. Apoptosis was detected using an Annexin V-FITC/PI double staining kit (BD Biosciences, Franklin Lakes, NJ, USA) according to the manufacturer's protocol. Cells were seeded at a concentration of $5 \times 10^{3}$ cells $/ 100 \mu \mathrm{l} /$ well in 96 -well culture plates, then $0.1 \mu \mathrm{M}$ gefitinib was added $48 \mathrm{~h}$ prior to detection. Cells were harvested and washed twice with cold PBS by gentle shaking. Cells were then re-suspended and added to Binding buffer (1X), and cell density was adjusted to $2-5 \times 10^{5} / \mathrm{ml}$. In the dark, $5 \mu \mathrm{l}$ Annexin V-FITC was added to the cell suspension volume of $195 \mu \mathrm{l}$ and incubated for $10 \mathrm{~min}$ at room temperature prior to the addition of $190 \mu \mathrm{l}$ binding buffer (1X) and $10 \mu \mathrm{l}$ propidium iodide (PI). A total of 10,000 events per sample were acquired using a FACS-scan flow cytometer (BD Biosciences, San Jose, CA, USA), and the percentage of cells undergoing apoptosis was analyzed using BD CellQuest ${ }^{\mathrm{TM}}$ Pro Software Analysis Tutorial (Version 5.1; BD Biosciences).

Cell cycle analysis. PC9-R cells seeded in 6-well plates ( $3 \times 10^{5} /$ well) were treated either with transfection of MIR31HG siRNA or a siRNA scramble control in an RPMI-1640 medium with $10 \%$ fetal bovine serum for cell cycle analyses, and $0.1 \mu \mathrm{M}$ gefitinib was added to the plates for $48 \mathrm{~h}$. The cells were then harvested and fixed in cold $70 \%$ ethanol. The samples were incubated with RNase A $(60 \mu \mathrm{g} / \mathrm{ml})$ and PI $(25 \mu \mathrm{g} / \mathrm{ml})$ for $20 \mathrm{~min}$ in the dark at room temperature. Samples were measured on a FACSCalibur flow cytometer (BD Biosciences), and cell cycle stages were analyzed using ModFit Software (version 3.2; Verity Software House Inc., Topsham, ME, USA).

lncRNA microarray. PC9 and PC9-R cells were used to synthesize double-stranded cDNA. Double-stranded cDNA was labeled and hybridized to the $8660 \mathrm{~K}$ LncRNA Expression Microarray (Array Star Inc., Rockville, MD, USA). After hybridization and washing, processed slides were scanned with the Agilent DNA Microarray Scanner (G2505B; Agilent Technologies, Inc., Santa Clara, CA, USA). Agilent Feature Extraction software ver. 10.7.3.1 (Agilent Technologies, Inc.) was used to analyze acquired array images. Quantile normalization and subsequent data processing were performed using the Gene Spring GX software package version 11.5.1 (Agilent Technologies, Inc.). Differentially expressed genes were identified through the random variance model. A P-value was calculated using the paired t-test. The threshold set for up and downregulated genes was a fold-change $\geq 2.0$ and a $P$-value $\leq 0.05$. Each cell line performed lncRNA microarray in triplicate.

Reverse transcription-quantitative polymerase chain reaction $(R T-q P C R)$. The differential genes were verified by using RT-qPCR. The specific operation steps are as follows: Total cellular RNA was isolated from the cultured PC9 and PC9-R cells using TRIzol reagent (Invitrogen; Thermo Fisher Scientific, Inc.) according to the protocol of the manufacturer. Total RNA was used to synthesize cDNA with PrimeScript $^{\mathrm{TM}}$ RT reagent kit (cat. no. RR037A; Takara Bio, Inc., Otsu, Japan), and subjected to qPCR. The relative expression levels of lncRNA were measured using a SYBR ${ }^{\circledR}$ Premix Ex Taq ${ }^{\mathrm{TM}}$ (cat. no. RR420A; Takara Bio, Inc.) following the protocol of the manufacturer with GADPH as an internal control. RT-qPCR primers were synthesized by Sangon Biotech Co., (Shanghai, China). The 5'-3' primer sequences used for RT-qPCR were as follows: PVT1 forward, GGA CGGACTTGAGAACTGT and reverse, GGCTTGTGAATC TGGGAG; H19 forward, GCACTAAGTCGATTGCACTGG and reverse, GCCTCAAGCAGACGGCCACA; MIR31HG forward, TCCCAGTTTCAGACCACC and reverse, CCA GGCTATGTCTTTCCTCTAT; CBR3-AS1 forward, ACA GCACGCATTCACCAG and reverse, TTGTAGCCGCCA AGTTTC; lincRNA-p21 forward, GGGTGGCTCACTCTT 
CTGGC and reverse, TGGCCTTGCCCGGGCTTGTC; GAPDH forward, GACTCATGACCACAGTCCATGC and reverse, AGAGGCAGGGATGATGTTCTG. For RT-qPCR, the reaction was performed on ABI 7500 Sequence Detection System (Applied Biosystems; Thermo Fisher Scientific, Inc.). The following cycles were used: $95^{\circ} \mathrm{C}$ for $30 \mathrm{sec}, 40$ cycles of $95^{\circ} \mathrm{C}$ for $5 \mathrm{sec}$ and $60^{\circ} \mathrm{C}$ for $31 \mathrm{sec}$. The dissociation stage was $95^{\circ} \mathrm{C}$ for $15 \mathrm{sec}, 60^{\circ} \mathrm{C}$ for $1 \mathrm{~min}$ and $95^{\circ} \mathrm{C}$ for $15 \mathrm{sec}$. The relative expression levels of target genes were calculated as $2^{-(\mathrm{Cq} \text { of target genes)-(Cq of GAPDH) }}$ (17) subsequent to normalization with reference to the quantification of GAPDH. The experiment was repeated three times.

Western blot analysis. PC9-R cells seeded in 6-well plates (3x10 $/$ well) were treated either with transfection MIR31HG siRNA or a si-scramble control for $48 \mathrm{~h}$. For the RNA interference-mediated knockdown of MIR31HG, three siRNAs for depletion of MIR31HG were synthesized and generated by GenePharma, Inc. (Sunnyvale, CA, USA). The sequence for MIR31HG siRNA was: siRNA-1, 5'-GCAAAGAAG UCCGAGGC-3'; siRNA-2, 5'-UCAAAGGACACGCCA AGUG-3'; siRNA-3, 5'-GAGAAGAAAGAAGUCACC-3'. Cells were harvested and lysed with immunoprecipitation buffer supplemented with $1 \mathrm{mM}$ sodium vanadate, $0.5 \mathrm{mM}$ dithiothreitol, $1 \mathrm{mM}$ phenylmethylsulfonyl fluoride, $2 \mathrm{mM}$ leupeptin, $2 \mathrm{mM}$ aprotinin, and $2 \mathrm{mM}$ pepstatin on ice for $30 \mathrm{~min}$. The total cellular protein content was determined using a Bio-Rad protein assay reagent (Bio-Rad Laboratories Inc., Hercules, CA, USA). Aliquots of the protein extracts were resolved by $10 \%$ SDS-PAGE and transferred to nitrocellulose (cat. no. HATF00010; EMD Millipore, Billerica, MA, USA) membranes. Membranes were blocked with 5\% nonfat dry milk in Tris-buffered saline and incubated with antibodies against p-EGFR (cat. no. PL-0302648; dilution, 1/1,000; PLLABS, Nanaimo, BC, Canada), total EGFR (cat. no. AB36836; dilution, 1:2,000; AbSci, Vancouver, BC, Canada), p-PI3K (cat. no. BS4605; dilution, 1:2,000; Bioworld Technology, Inc., St. Louis Park, MN, USA), total PI3K (cat. no. NB100-75198; dilution, 1:1,500; Novus Biologicals, Inc., Abingdon, UK), p-AKT (cat. no. xyP001a; dilution, 1:3,000; Abcam, Cambridge, UK), total AKT (cat. no. AB27174; dilution, 1:10,000; AbSci), phosphorylated mouse minute 2 homolog (p-Mdm2; cat. no. US1506136; dilution, 1:500; Merck \& Co., Inc., Whitehouse Station, NJ, USA), P53 (cat. no. 1026-1; dilution, 1:2,000: Epitomics, Burlingame, CA, USA), GAPDH (cat. no. PA116777; dilution, 1:2,000: Thermo Fisher Scientific, Inc.), Caspase-3 (cat. no. 1087-1; dilution, 1:1,000; Epitomics), Caspase-9 (cat. no. DB081; dilution, 1:5,000; Acris Antibodies GmbH; OriGene, Herford, Germany), Bax (cat. no. PA112602; dilution, 1:1,000; Thermo Fisher Scientific, Inc.) and Bcl-2 (cat. no. MA126233; dilution, 1:1,000; Thermo Fisher Scientific, Inc.) at $4^{\circ} \mathrm{C}$ overnight. The membranes were washed and subsequently probed with secondary antibody, goat anti-mouse IgG conjugated to horseradish peroxidase (Santa Cruz Biotechnology, Inc., Dallas, TX, USA) at a dilution of 1:4,000 for $1 \mathrm{~h}$ at room temperature. Proteins were visualized with a chemiluminescence reagent (Pierce; Thermo Fisher Scientific, Inc.). GAPDH was used as the internal control.
Statistical analysis. SPSS version 19.0 for Windows (IBM SPSS, Armonk, NY, USA) was used for all analyses. Student's t-test was used to compare the differences between groups. P-values were based on the two-sided statistical analysis and $\mathrm{P}<0.05$ was considered to indicate a statistically significant difference.

\section{Results}

Sensitivity of PC9 and PC9-R cells to gefitinib. The $\mathrm{IC}_{50}$ values of gefitinib in PC9 and PC9-R cells treated with various concentration of the drug ranging from $0.01-80 \mu \mathrm{M}$ for $48 \mathrm{~h}$ were assessed. As expected, PC9-R cells were resistant to gefitinib $\left(\mathrm{IC}_{50}=8.1 \mu \mathrm{M}\right)$, and PC9 cells were sensitive to gefitinib $\left(\mathrm{IC}_{50}=0.115 \mu \mathrm{M}\right.$; Fig. 1A).

Rates of apoptosis of PC9 and PC9-R cells following gefitinib treatment. The rates of apoptosis of PC9 and PC9-R cells treated for $48 \mathrm{~h}$ in $0.1 \mu \mathrm{M}$ gefitinib were analyzed by flow cytometry. The rate of apoptosis in PC9 cells markedly increased $(\mathrm{P}<0.001)$, but in PC9-R cells it did not (Fig. 1B).

Microarray of PC9 and PC9-R cell lines. A gene chip study was performed using the Array Star probe dataset (Array Star, Inc.) to investigate possible lncRNA expression changes that may cause gefitinib resistance. Hierarchical clustering showed systematic variations in the expression of lncRNAs between PC9 and PC9-R (Fig. 2A).

Validation of the microarray data using $R T-q P C R$. To validate the microarray analysis findings, the expression levels of lncRNAs in both PC9 and PC9-R cell lines were analyzed using RT-qPCR. The expression of PVT1, H19, MIR31HG, BOK-AS1, CBR3-AS1 and LincRNA-p21 differed, and PVT1 ( $\mathrm{P}=0.0058)$, MIR31HG $(\mathrm{P}<0.001)$ and LincRNA-p21 $(\mathrm{P}=0.0036)$ were significantly decreased, especially expression of MIR31HG, which was ten times higher in PC9-R than in PC9 (Fig. 2B).

MIR31HG lncRNAs knockdown sensitizes PC9-R cells to gefitinib. The effect of MIR31HG lncRNA knockdown on PC9-R sensitivity to gefitinib was investigated. Knockdown was carried out by designing and then transfecting siRNA targeting MIR31HG into PC9-R cells. MIR31HG lncRNA expression in the transfected cells was measured by RT-qPCR. As expected, MIR31HG lncRNA expression levels were decreased $(\mathrm{P}<0.001)$ in the cells containing si-MIR31HG, compared to those transfected with a si-scramble control (Fig. 3A). To determine the sensitivity of si-MIR31HG of PC9-R cells to gefitinib, the CCK-8 cell viability assay (Dojindo Molecular Technologies, Inc.) was used to measure the effect of gefitinib on the cells transfected with si-MIR31HG, and both PC9 and PC9-R cells. The results of the cell viability assay demonstrated that knockdown of MIR31HG lncRNA in PC9-R cells significantly increases their sensitivity to gefitinib $\left(\mathrm{IC}_{50}, 0.93 \mu \mathrm{M}\right)$, compared with PC9-R cells $\left(\mathrm{IC}_{50}, 8.1 \mu \mathrm{M}\right), \mathrm{P}<0.001$. Indeed, they exhibit sensitivity to gefitinib comparable with PC9 cells $\left(\mathrm{IC}_{50}, 0.115 \mu \mathrm{M}\right.$; Fig. 3B). In addition, the rate of apoptosis in PC9-R cells transfected with si-MIR31HG IncRNA was $12.3 \%$ and significantly increased, compared with PC9-R cells 
A

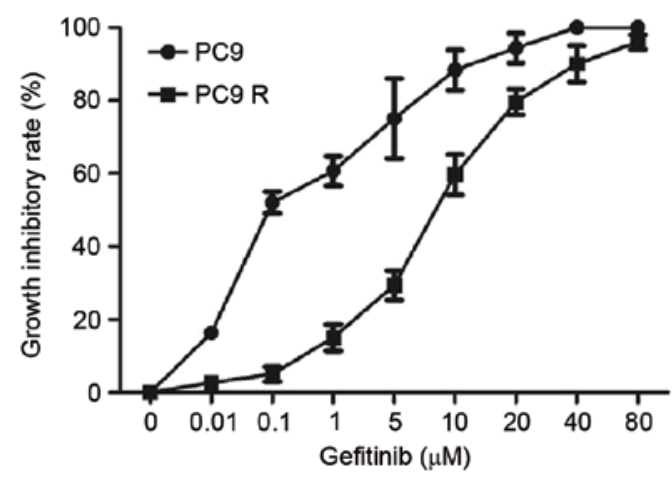

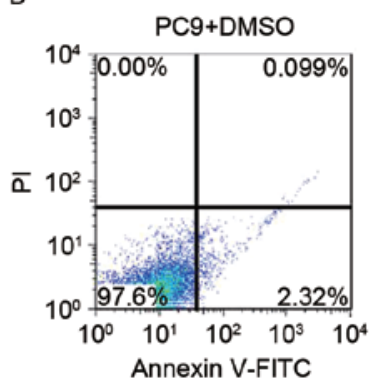

PC9-R+DMSO

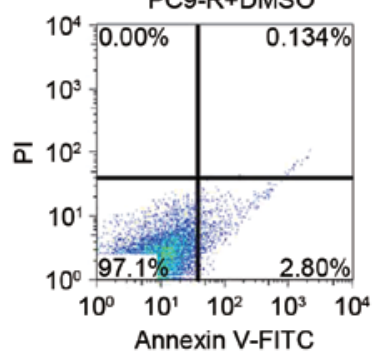

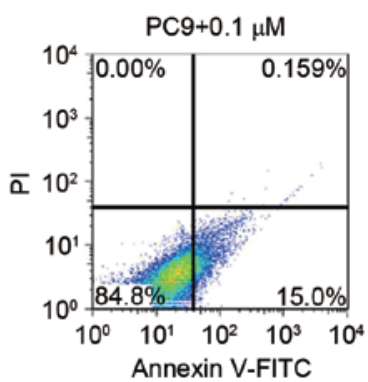

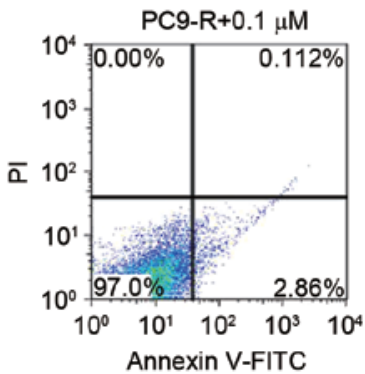

Figure 1. Cell growth inhibition and apoptosis in PC9 and PC9-R cells following treatment with gefitinib. (A) Cells from the PC9 and PC9-R cell lines were treated with gefitinib for $48 \mathrm{~h}$, and cell proliferation was measured using the Cell Counting Kit-8 assay. PC9-R cells exhibited resistance to gefitinib $\left(\mathrm{IC}_{50}=8.1 \mu \mathrm{M}\right)$, and PC9 cells exhibited sensitivity $\left(\mathrm{IC}_{50}=0.115 \mu \mathrm{M}\right)$. (B) As expected, gefitinib increased the rate of apoptosis in PC9 cells, but did not in PC9-R cells.

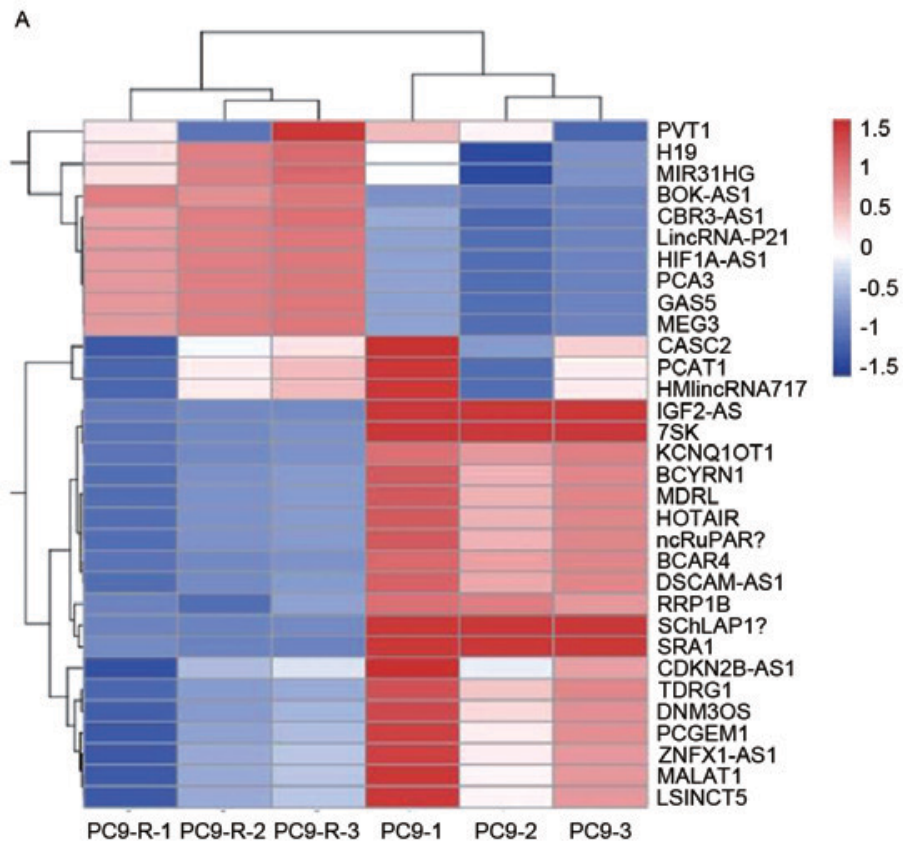

Figure 2. PC9 and PC9-R cells have differential expression of lncRNAs. (A) Heat maps of lncRNAs profiles in PC9-R from PC9 were compared. Each sample was assayed in triplicate. Both down-regulated (blue) and up-regulated (red) lncRNAs were identified in PC9 and PC9-R cells. (B) Microarray data was validated by RT-qPCR. The expression of PVT1, H19, MIR31HG, BOK-AS1, CBR3-AS1 and LincRNA-P21 were significantly different between the two cell lines, especially MIR31HG expression. lncRNA, long noncoding RNA.

and those transfected with a si-scramble control (Fig. 3C), respectively were 2.9 and $3.0 \%(\mathrm{P}<0.001)$.

MIR31HG lncRNAs knockdown sensitizes PC9-R cells to gefitinib. To delineate the molecular mechanism involved in the MIR31HG lncRNA knockdown sensitization of PC9-R cells to gefitinib, western blotting was used to analyze the changes in protein levels of the key components of the
EGFR/PI3K/AKT signaling pathways in PC9-R cells transfected with si-MIR31HG. The western blot analysis indicated that knockdown of MIR31HG IncRNA significantly reduces the expression of $\mathrm{p}-\mathrm{EGFR}(\mathrm{P}=0.0027)$, $\mathrm{p}-\mathrm{PI} 3 \mathrm{~K}(\mathrm{P}=0.0016)$, $\mathrm{p}-\mathrm{AKT}$ $(\mathrm{P}=0.0003)$ and $\mathrm{p}-\mathrm{Mdm}-2(\mathrm{P}=0.0003)$ proteins in $\mathrm{PC}$ - $\mathrm{R}$ cells, compared to untreated and si-scramble cells, but does not alter total EGFR, PI3K or AKT levels. It also activates expression of p53 (Fig. 4A). This indicates that transfection of PC9-R 
A

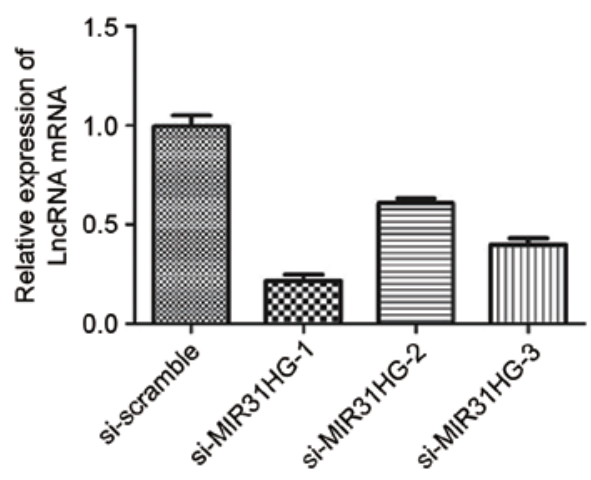

B

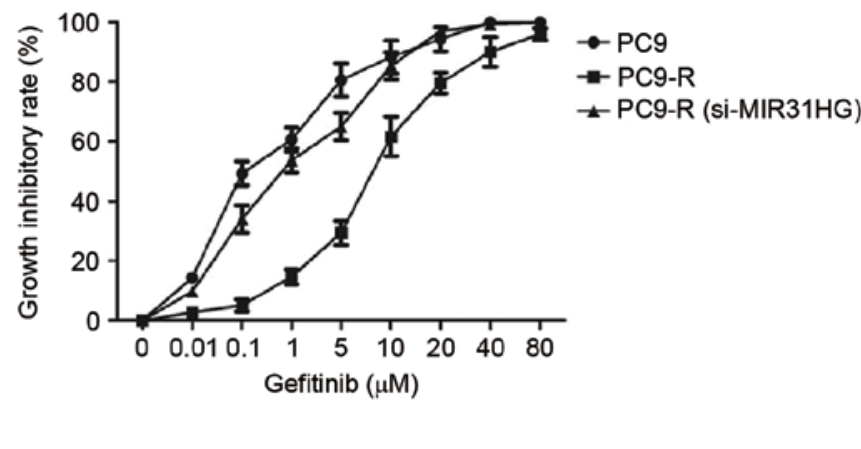

C
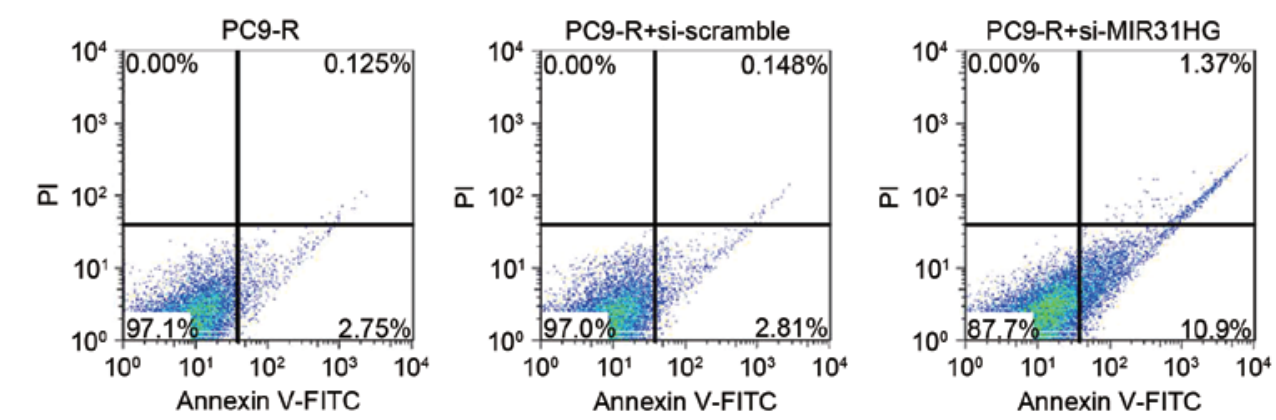

Figure 3. RT-qPCR analysis of MIR31HG knockdown using siRNA. (A) RT-qPCR was used to examine expression levels of MIR31HG lncRNAs in the transfection cells. (B) PC9, PC9-R and PC9-R cells with si-MIR31HG were treated with gefitinib for $48 \mathrm{~h}$, and the cell viability was measured using the Cell Counting Kit-8 assay. PC9-R cells transfected with si-MIR31HG exhibited an increased sensitivity to gefitinib $\left(\mathrm{IC}_{50}=0.93 \mu \mathrm{M}\right)$, compared to PC9-R cells (C) The rate of apoptosis in PC9-R cells transfected with si-MIR31HG significantly increased, but in PC9-R cells and PC9-R cells transfected with si-scramble it did not. RT-qPCR, reverse transcription-polymerase chain reaction; lncRNA, long noncoding RNA.

with si-MIR31HG turns off the EGFR/PI3K/AKT signaling pathway. Furthermore, the western blot analysis showed that PC9-R cells transfected with si-MIR31HG increased the expression of proteins involved in the cell mitochondrial apoptosis pathway. Compared with expression levels in the control group, Caspase-3 ( $\mathrm{P}=0.0031)$, Caspase-9 $(\mathrm{P}=0.0047)$ and $\mathrm{Bax}(\mathrm{P}<0.001)$ were significantly increased, however Bcl-2 $(\mathrm{P}<0.001)$ expression was repressed (Fig. 4B), demonstrating that transfection of PC9-R cells with si-MIR31HG induces cell apoptosis by activating the mitochondrial apoptosis pathway.

Cell cycle distribution of PC9-R cells following knockdown by MIR31HG IncRNAs. The effect of si-MIR31HG on the cell cycle was analyzed by a FACS Calibur flow cytometer (BD Biosciences), which showed that a higher percentage of cells in the si-MIR31HG group were in the G0/G1 phase than in the PC9-R cells group and those transfected with si-scramble group, the P-values were 0.0189 and 0.016 , respectively (Fig. 4C). The si-MIR31HG group also contained a lower percentage of cells in the $\mathrm{S}$ phase compared with other groups, $\mathrm{P}$-values were 0.0264 and 0.0307 respectively (Fig. 4C). These results indicate that PC9-R cells transfected with si-MIR31HG are able to arrest the cell cycle in the G0/G1 phase.

\section{Discussion}

Gefitinib has proven to be highly effective at treating advanced or metastatic NSCLC in patients harboring an activating
EGFR mutation, which competes with ATP for binding to the tyrosine kinase pocket of the receptor (18). Compared with conventional chemotherapy and radiotherapy, EGFR-TKI drugs have fewer side effects and significantly longer progression free survival rates for patients with activating EGFR mutations $(19,20)$. However, many patients treated with gefitinib later develop resistance to the drug. The T790M mutation and MET amplification are well-studied mechanisms for acquired gefitinib resistance $(21,22)$.

IncRNAs are long non-protein coding RNAs whose dysregulation is related to prognosis and chemotherapy resistance in human cancers (23-26). Various studies have suggested that the dysregulation of IncRNA contributes to lung cancer with lymph node metastasis, advanced stage, metastasis development and poor patient prognosis (11-16,27,28). Cheng et al (29) have analyzed EGFR-TKI-sensitive and EGFR-TKI-resistant human lung cancer cells by lncRNA microarray. Their results suggested that numerous IncRNAs were differentially expressed in gefitinib-sensitive and gefitinib-resistant PC9 cells. However, the exact mechanism by which differentially expressed IncRNAs are correlated with EGFR-TKI resistance remained unknown.

The present study identified differentially expressed lncRNAs in PC9 and PC9-R cells by microarray and RT-qPCR . The results indicated that levels of expression of PVT1, H19, MIR31HG, BOK-AS1, CBR3-AS1 and LincRNA-P21 differed significantly between the two cell lines, in particular the expression of MIR31HG. Following this, the molecular mechanism involved in EGFR-TKIs resistance in NSCLC was delineated 
A

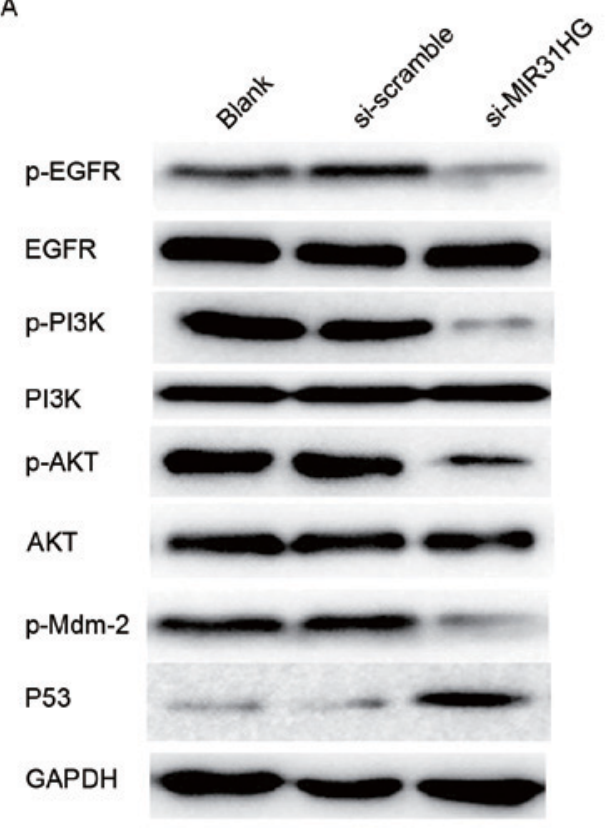

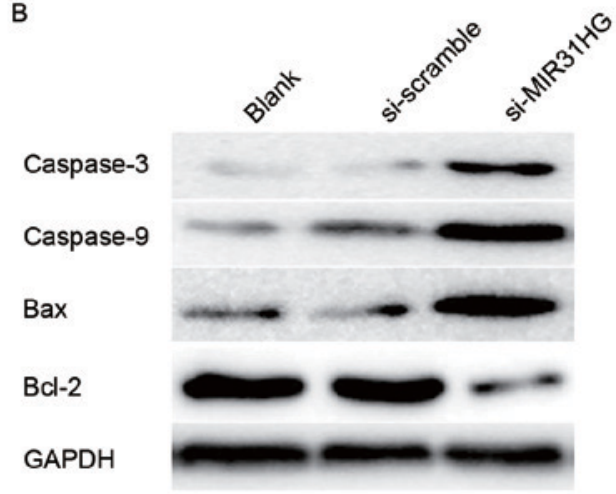

C

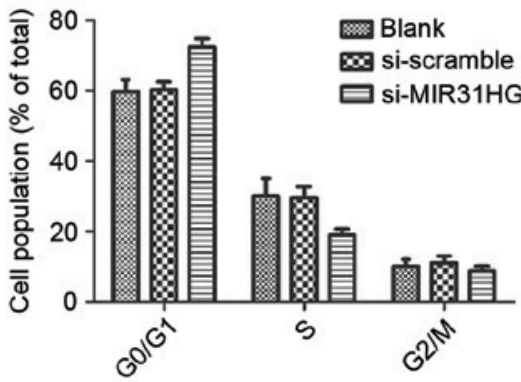

Figure 4. Knockdown of MIR31HG alters protein expression and cell cycle distribution in PC9-R cells. (A) Western blot analysis revealed that PC9-R cells transfected with si-MIR31HG repressed p-EGFR, p-PI3K, p-AKT and p-Mdm-2 expression, but did not alter total EGFR, PI3K or AKT levels. It also stimulated expression of p53. (B) The result showed that PC9-R cells containing si-MIR31HG increased expression of the proteins Caspase-3, Caspase-9 and Bax, but repressed Bcl-2, compared to levels in the control group. (C) The effect of si-MIR31HG on cell cycle was analyzed by flow cytometry. This showed that PC9-R cells transfected with si-MIR31HG were able to arrest the cell cycle at the G0/G1 phase. EGFR, epidermal growth factor receptor; PI3K, phosphatidylinositol-3 kinase; AKT, protein kinase B; p-EGFR, phosphorylated epidermal growth factor receptor; p-I3K, phosphorylated phosphatidylinositol-3 kinase; p-AKT, phosphorylated protein kinase B; GAPDH, glyceraldehyde 3-phosphate dehydrogenase.

using a CCK-8 cell viability assay to determine the sensitivity of PC9-R cells transfected with si-MIR31HG to gefitinib. Western blotting was carried out to monitor the changes in protein levels of key components of EGFR/PI3K/AKT signaling pathways involved.

A number of previous studies have demonstrated that the activation of PI3K/AKT and MEK/ERK cell signaling pathways is associated with EGFR TKI resistance in NSCLC (30,31). Kang et al (32) have reported that bufalin inhibits cell proliferation and induces cell apoptosis by inhibiting the MET/PI3K/AKT pathway and activating death-signaling pathways. PI3K/AKT is an important downstream signaling cascade of EGFR, which is overexpressed in NSCLC (33). Dysregulation of PI3K/AKT signaling pathways is related to reduced rates of apoptosis and the phenotype of multidrug resistance (34).

In the present study, PC9-R cells transfected with si-MIR31HG lncRNA exhibited an increased sensitivity to gefitinib and a higher rate of apoptosis. The si-MIR31HG PC9-R cells also had a reduced expression of $\mathrm{p}-\mathrm{EGFR}, \mathrm{p}-\mathrm{PI} 3 \mathrm{~K}$, $\mathrm{p}-\mathrm{AKT}$ and $\mathrm{p}-\mathrm{Mdm}-2$ proteins, and increased expression of p53. Total levels of EGFR, PI3K and AKT remained the same. Mdm-2 has been identified as a protein that represses p53 transcriptional activity and so its reduced expression in si-MIR31HG PC9-R cells may increase p53 expression, which initiates cell apoptosis and regulates the cell cycle (35). Therefore, inhibition of EGFR/PI3K/AKT pathway could decrease cell proliferation and promote apoptosis by increasing levels of $\mathrm{p} 53$.
Mitochondrial integrity is central to both caspase-dependent and -independent cell death. Regulation of the mitochondrial pathway is under the control of the Bcl-2 family, which includes pro-apoptotic proteins such as Bax, Bad, and Bak, and anti-apoptotic proteins, such as Bcl-2, Bcl-XL, and Bcl-W (36). The mitochondrial pathway is activated by the release of cytochrome $c$, which is followed by caspase- 9 and caspase-3 activation (7,37). The current study demonstrated that PC9-R cells transfected with si-MIR31HG lncRNAs expressed significantly higher levels of Caspase-3, Caspase-9 and Bax proteins, but reduced levels of Bcl-2. This has demonstrated for the first time that PC9-R cells transfected with si-MIR31HG exert pro-apoptotic function via the mitochondrial pathway by inhibiting the EGFR/PI3K/AKT pathway. Furthermore, regulation of the cell cycle is vital to regulate cell growth, and some proteins or chemical compounds could trigger apoptosis in tumor cells accompanied by cell arrest (38). The present study demonstrated that PC9-R cells transfected with si-MIR31HG were able to arrest the cell cycle in $\mathrm{G} 0 / \mathrm{G} 1$ phase, thus regulating the cell cycle.

In conclusion, PC9-R cells transfected with si-MIR31HG developed enhanced sensitivity to gefitinib by inhibiting the EGFR/PI3K/AKT pathway and activating p53. They also induced cell apoptosis via activation of the mitochondrial apoptosis pathway leading to arrest of the cell cycle in the G2/M phase. Therefore, over-expression of MIR31HG lncRNA contributes to gefitinib resistance in the PC9-R cell, by affecting cell proliferation, apoptosis and the cell cycle through activation of the EGFR/PI3K/AKT pathway. 


\section{Acknowledgements}

The present study was supported by grants from the National Nature Science Foundation of China (grant no. 81301927), Zhejiang Provincial Nature Science Foundation (grant no. LY13H160023) and Hangzhou Medical Major Disease Project (grant no. 20130733Q03).

\section{References}

1. Siegel R, Ma J, Zou Z and Jemal A: Cancer statistics, 2014. CA Cancer J Clin 64: 9-29, 2014.

2. Dela Cruz CS, Tanoue LT and Matthay RA: Lung cancer: Epidemiology, etiology, and prevention. Clin Chest Med 32: 605-644, 2011.

3. Hotta K, Kiura K, Ueoka H, Tabata M, Fujiwara K, Kozuki T, Okada T, Hisamoto A and Tanimoto M: Effect of gefitinib ('Iressa', ZD1839) on brain metastases in patients with advanced non-small-cell lung cancer. Lung Cancer 46: 255-261, 2004.

4. Burotto M, Manasanch EE, Wilkerson J and Fojo T: Gefitinib and erlotinib in metastatic non-small cell lung cancer: A meta-analysis of toxicity and efficacy of randomized clinical trials. Oncologist 20: 400-410, 2015.

5. Engelman JA and Settleman J: Acquired resistance to tyrosine kinase inhibitors during cancer therapy. Curr Opin Genet Dev 18: 73-79, 2008

6. Prabhakar CN: Epidermal growth factor receptor in non-small cell lung cancer. Transl Lung Cancer Res 4: 110-118, 2015.

7. Chen J, Wang W, Wang H, Liu X and Guo X: Combination treatment of ligustrazine piperazine derivate DLJ14 and adriamycin inhibits progression of resistant breast cancer through inhibition of the EGFR/PI3K/Akt survival pathway and induction of apoptosis. Drug Discov Ther 8: 33-41, 2014.

8. Ørom UA, Derrien T, Beringer M, Gumireddy K, Gardini A, Bussotti G, Lai F, Zytnicki M, Notredame C, Huang Q, et al: Long noncoding RNAs with enhancer-like function in human cells. Cell 143: 46-58, 2010.

9. Cui Z, Ren S, Lu J, Wang F, Xu W, Sun Y, Wei M, Chen J, Gao X, $\mathrm{Xu} \mathrm{C}$, et al: The prostate cancer-up-regulated long noncoding RNA PlncRNA-1 modulates apoptosis and proliferation through reciprocal regulation of androgen receptor. Urol Oncol 31: $1117-1123,2013$.

10. Yang F, Zhang L, Huo XS, Yuan JH, Xu D, Yuan SX, Zhu N, Zhou WP, Yang GS, Wang YZ, et al: Long noncoding RNA high expression in hepatocellular carcinoma facilitates tumor growth through enhancer of zeste homolog 2 in humans. Hepatology 54: 1679-1689, 2011.

11. Ji P, Diederichs S, Wang W, Böing S, Metzger R, Schneider PM, Tidow N, Brandt B, Buerger H, Bulk E, et al: MALAT-1, a novel noncoding RNA and thymosin beta4 predict metastasis and survival in early-stage non-small cell lung cancer. Oncogene 22: 8031-8041, 2003.

12. Liu XH, Liu ZL, Sun M, Liu J, Wang ZX and De W: The long non-coding RNA HOTAIR indicates a poor prognosis and promotes metastasis in non-small cell lung cancer. BMC Cancer 13: 464, 2013.

13. Sun M, Liu XH, Wang KM, Nie FQ, Kong R, Yang JS, Xia R, $\mathrm{Xu}$ TP, Jin FY, Liu ZJ, et al: Down regulation of BRAF activated non-coding RNA is associated with poor prognosis for non-small cell lung cancer and promotes metastasis by affecting epithelialmesenchymal transition. Mol Cancer 13: 68, 2014.

14. Han L, Kong R, Yin DD, Zhang EB, Xu TP, De W and Shu YQ: Low expression of long noncoding RNA GAS6-AS1 predicts a poor prognosis in patients with NSCLC. Med Oncol 30: 694, 2013.

15. Zhang L, Zhou XF, Pan GF and Zhao JP: Enhanced expression of long non-coding RNA ZXF1 promoted the invasion and metastasis in lung adenocarcinoma. Biomed Pharmacother 68: 401-407, 2014.

16. Ricciuti B, Mecca C, Crinò L, Baglivo S, Cenci M and Metro G: Non-coding RNAs in lung cancer. Oncoscience 1: 674-705, 2014

17. Thomas D and Livak KJ: Analyzing real-time PCR data by the comparative C(T) method. Nat Protoc 3: 1101-1108, 2008.

18. Rahman AF, Korashy HM and Kassem MG: Gefitinib. Profiles Drug Subst Excip Relat Methodol 39: 239-264, 2014.
19. Pilkington G, Boland A, Brown T, Oyee J, Bagust A and Dickson R: A systematic review of the clinical effectiveness of first-line chemotherapy for adult patients with locally advanced or metastatic non-small cell lung cancer. Thorax 70: 359-367, 2015.

20. Yuan Y, Li XF, Chen JQ, Dong CX, Weng SS and Huang JJ: Critical appraisal of the role of gefitinib in the management of locally advanced or metastatic non-small cell lung cancer. Onco Targets Ther 7: 841-852, 2014.

21. Engelman JA and Jänne PA: Mechanisms of acquired resistance to epidermal growth factor receptor tyrosine kinase inhibitors in non-small cell lung cancer. Clin Cancer Res 14: 2895-2899, 2008.

22. Engelman JA, Zejnullahu K, Mitsudomi T, Song Y, Hyland C, Park JO, Lindeman N, Gale CM, Zhao X, Christensen J, et al: MET amplification leads to gefitinib resistance in lung cancer by activating ERBB3 signaling. Science 316: 1039-1043, 2007.

23. Zhang J, Zhu $\mathrm{N}$ and Chen X: A novel long noncoding RNA LINC01133 is upregulated in lung squamous cell cancer and predicts survival. Tumour Biol 36: 7465-7471, 2015.

24. Cai H, Chen J, He B, Li Q, Li Y and Gao Y: A FOXM1 related long non-coding RNA contributes to gastric cancer cell migration. Mol Cell Biochem 406: 31-41, 2015.

25. Gao Y, Chen G, Zeng Y, Zeng J, Lin M, Liu X and Liu J: Invasion and metastasis-related long noncoding RNA expression profiles in hepatocellular carcinoma. Tumour Biol 36: 7409-7422, 2015.

26. Ren S, Wang F, Shen J, Sun Y, Xu W, Lu J, Wei M, Xu C, Wu C, Zhang Z, et al: Long non-coding RNA metastasis associated in lung adenocarcinoma transcript 1 derived miniRNA as a novel plasma-based biomarker for diagnosing prostate cancer. Eur J Cancer 49: 2949-2959, 2013.

27. Qiu M, Xu Y, Yang X, Wang J, Hu J, Xu L and Yin R: CCAT2 is a lung adenocarcinoma specific long non-coding RNA and promotes invasion of non-small cell lung cancer. Tumour Biol 35: 5375-5380, 2014

28. Gutschner T, Hämmerle M, Eissmann M, Hsu J, Kim Y, Hung G, Revenko A, Arun G, Stentrup M, Gross M, et al: The noncoding RNA MALAT1 is a critical regulator of the metastasis phenotype of lung cancer cells. Cancer Res 73: 1180-1189, 2013.

29. Cheng N, Li X, Zhao C, Ren S, Chen X, Cai W, Zhao M, Zhang Y, Li J, Wang Q and Zhou C: Microarray expression profile of long non-coding RNAs in EGFR-TKIs resistance of human non-small cell lung cancer. Oncol Rep 33: 833-839, 2015.

30. Nguyen KS, Kobayashi S and Costa DB: Acquired resistance to epidermal growth factor receptor tyrosine kinase inhibitorsin non-small-cell lung cancers dependent on the epidermal growth factor receptor pathway. Clin Lung Cancer 10: 281-289, 2009.

31. Li H, Schmid-Bindert G, Wang D, Zhao Y, Yang X, Su B and Zhou C: Blocking the PI3K/AKT and MEK/ERK signaling pathways can overcome gefitinib-resistance in non-small cell lung cancer cell lines. Adv Med Sci 56: 275-284, 2011.

32. Kang XH, Xu ZY, Gong YB, Wang LF, Wang ZQ, Xu L, Cao F and Liao MJ: Bufalin reverses HGF-induced resistance to EGFR-TKIs in EGFR mutant lung cancer cells via blockage of Met/PI3k/Akt pathway and induction of apoptosis. Evid Based Complement Alternat Med 2013: 243859, 2013.

33. Sordella R, Bell DW, Haber DA and Settleman J: Gefitinib-sensitizing EGFR mutations in lung cancer activate anti-apoptotic pathways. Science 305: 1163-1167, 2004.

34. Qiao M, Sheng S and Pardee AB: Metastasis and AKT activation. Cell Cycle 7: 2991-2996, 2008.

35. Slack A, Chen Z, Tonelli R, Pule M, Hunt L, Pession A and Shohet JM: The p53 regulatory gene MDM2 is a direct transcriptional target of MYCN in neuroblastoma. Proc Natl Acad Sci USA 18: 731-736, 2005.

36. Harris $\mathrm{MH}$ and Thompson CB: The role of the Bcl-2 family in the regulation of outer mitochondrial membrane permeability. Cell Death Differ 7: 1182-1191, 2000.

37. Elkholi R, Renault TT, Serasinghe MN and Chipuk JE: Putting the pieces together: How is the mitochondrial pathway of apoptosis regulated in cancer and chemotherapy? Cancer Metab 2: 16, 2014.

38. Muthu M, Cheriyan VT and Rishi AK: CARP-1/CCAR1: A biphasic regulator of cancer cell growth and apoptosis. Oncotarget 6: 6499-6510, 2015. 